\title{
Prognostic Significance of Heat Shock Protein 90 in Non Muscle Invasive Bladder Cancer Treated by BCG Immunotherapy
}

Faouzia Ajili ${ }^{1 *}$, Amel Nedri' ${ }^{1}$ Nadia Kourda ${ }^{2}$, Afifa maaloul ${ }^{1}$, salma Karay ${ }^{1}$, Amine Darouiche $^{3}$, Mouhamed Chebil ${ }^{3}$ and Samir Boubaker $^{1}$

${ }^{1}$ Laboratory of Human and Experimental Pathology, Institute Pasteur of Tunis, Tunisia

${ }^{2}$ Departmenty of Pathology, Charles Nicolle Hospital, Tunis, Tunisia

${ }^{3}$ Departmenty of Urology, Charles Nicolle Hospital, Tunis, Tunisia

\begin{abstract}
Background: The heat shock protein 90 (HSP90) is overexpressed and highly associated with poor prognosis in many malignancies. However, the role of HSP90 in Non muscle invasive bladder cancer (NMIBC) has not been thoroughly elucidated The aim of this study is to investigate the relationship of HSP90 expression with the response to BCG immunotherapy of NMIBC.

Methods: HSP90 expression was evaluated by immunohistochemistry in 43NMIBC samples. The relationships between HSP90 and the response to BCG immunotherapy were analyzed. The results were correlated with clinicopathological characteristics using univariate and multivariate analyses.
\end{abstract}

Results: Univariate Cox regression analysis of baseline characteristics and HSP90 expression showed that no significant association was found with BCG immunotherapy response $(p=0.894)$. In the other hand, multivariate Cox regression analysis showed that HSP90 protein is not an independent factor of tumor response after BCG immunotherapy $(p=0.884)$.

Conclusion: This study demonstrates that HSP90 could not be a useful prognostic marker for BCG treatment in NMIBC. We emphasize that this was a preliminary study and therefore further confirmation on a larger set of tissues is necessary.

Keywords: Bladder cancer; BCG immunotherapy; HSP90; Recurence; Prognostic

\section{Introduction}

Non-muscle-invasive bladder cancer (NMIBC) treated by transurethral surgery has high recurrence potential $(60-70 \%$ of cases) and a risk of invading muscle in $15-25 \%$ of patients [1]. Intravesical instillations of Bacillus Calmette-Guerin (BCG) are the most effective adjuvant treatment for preventing recurrence and progression [2]. The failure of this local immunotherapy requires a more aggressive surgery. Therefore, the primary treatment of choice still remains debatable, and it is not known whether early stage cystectomy or BCG therapy will be required. For these patients, the challenge is to recommend the correct treatment without delay, which will directly depend on risk progression. Thus, the assessment of the factors correlated with high risk of tumor recurrence after BCG therapy is an important step guiding the therapeutic protocol in NMIBC $[3,4]$. Currently there is no wellestablished predictive factor to evaluate BCG therapy response and the mechanism of the anti-tumor effect of BCG therapy is not very clear.

A biologic marker could help predict failure in refractory patients and allow early cystectomy before aggressive superficial carcinoma occurs. In fact, a delay in appropriate therapy may lead to metastatic dissemination and death. Recently, Heat Shock Proteins 90 (HSP90), have been proposed as prognostic factors for bladder cancer. These proteins are an important molecular chaperone for protein folding, intracellular disposition and proteolytic turnover of key regulators of cell growth and survival. They are up-regulated in response to cellular stresses or toxic insults that increase the degree of protein misfolding or aggregation and threaten cell survival $[5,6]$. As these stresses are characteristic of the tumor cell environment, the upregulation of heat shock proteins is commonly observed in cancer cells and is believed to support malignant transformation [7-9].

HSP90 is expressed in normal urothelium and decreased expression has been detected particularly in invasive bladder transitional cell carcinoma. Previous studies have also demonstrated an important correlation between the loss of HSP90 expression and poor prognosis, indicating a potential role for this protein in monitoring immune responses [10]. The role of HSP function in oncology is ubiquitous; intracellularly HSPs have been implicated in the regulation of apoptosis, whereas surface expression is associated in antibody assembly and antigen presentation [11,12]. In fact, these proteins are among the most immunogenic reported molecules $[13,14]$. Because BCG therapy is immune dependent, the activation of a Th1 immune response could be required for clinical efficacy; therefore, the role of HSPs in humoral response stimulated by BCG warrants investigation. The aim of this study is to investigate the prognostic and predictive value of HSP90 immunohistochemical expressions in a series of NMIBC patients before treatment, and to assess its correlation with BCGimmunotherapy response.

\section{Materials and Methods}

\section{Cohort of patients}

Our study includes 43 patients enrolled between 2006 and 2008 at the Department of Urology in Charles Nicolle Hospital, Tunis, Tunisia. Inclusion criteria were cystoscopically and histologically confirmed NMIBC including Ta and T1 stages. No cases of carcinoma in situ were found in our study. Other exclusion criteria were upper urinary tract tumors, urinary tract infections, and other neoplastic diseases. All patients were informed about the aim of the study and signed a written consent stating their agreement to participate in the

*Corresponding author: Ajili Faouzia, Laboratory of Human and Experimental Pathology, Pasteur Institute of Tunis, Tunisia. Institut Pasteur de Tunis 13, Place Pasteur, 1002 Tunis, Tunisia, Tel: 0021671789 608; Fax: 0021671791 833; E-mail: Faouziaagili@yahoo.fr

Received February 28, 2014; Accepted March 20, 2014; Published March 22, 2014

Citation: Ajili F, Nedri A, Kourda N, Maaloul A, Karay S, et al. (2014) Prognostic Significance of Heat Shock Protein 90 in Non Muscle Invasive Bladder Cancer Treated by BCG Immunotherapy. J Cytol Histol 5: 230. doi:10.4172/21577099.1000230

Copyright: (C) 2014 Ajili F, et al. This is an open-access article distributed under the terms of the Creative Commons Attribution License, which permits unrestricted use, distribution, and reproduction in any medium, provided the original author and source are credited. 
trial. The present study was also approved by the local research ethical committee of the Pasteur Institute of Tunis. The initial tumor, sampled immediately before BCG treatment was used for this purpose. Patients underwent complete transurethral resection, and the muscle layer was always assessable. Transitional cell carcinomas of the bladder were treated for the first time with 6 weekly instillations of intravesical BCG (BCG Pasteur strain, $75 \mathrm{mg}$ in $50 \mathrm{~mL}$ saline), 3-6 weeks after the last transurethral resection. After the last instillation, urinary cytology and cystoscopic examination with randomized sites of mapping cold biopsies were performed. If these examinations were negative, patients continued the maintenance treatment, which consists of 24 additional monthly instillations. The follow- up was performed for 26 months. The follow-up time was calculated as the number of months from the date of the surgical procedure to the last cystoscopical control or the last visit. Patients underwent urine cytology and cystoscopy every 3 months during the follow-up. Responders to BCG immunotherapy were defined as patients who did not show cystoscopic or cytological evidence of tumor recurrence during the 26 months. All patients were informed about the aim of the study and signed a written consent stating their agreement to participate in the trial. The present study was also approved by the local research ethical committee of the Pasteur Institute of Tunis which is in agreement with Helsinki declaration. Recurrence was defined as reappearance of tumor after the initial treatment with at least one tumor-free cystoscopy interval. The endpoint for follow-up was either development of recurrent cancer tumor or the termination date of the study. Follow-up results were recorded and used for univariate and multivariate analyses.

\section{Clinical and histological data}

The resulting grade and stage were evaluated according to the 2004 WHO grading system [15] and TNM 2002 revision. For each patient, data were collected on tumor size, number of tumor loci, histological grade (low grade and high grade), and stage (pTa or pT1).

\section{Immunohistochemistry}

The specimens for immunohistochemical analysis were formalinfixed and paraffin-embedded. One 4-mm section from each patient was cut and put on silanized microscope slides (Dako, Copenhagen, Denmark). Tissues sections were deparaffinized, and heat-induced antigen retrieval was performed in $0.01 \mathrm{M}$ citrate buffer, $\mathrm{pH}$ 6.0. They were then treated with $0.03 \%$ hydrogen peroxidase for $5 \mathrm{~min}$ to block endogenous peroxidase activity. They were washed 3 times in PBS for $5 \mathrm{~min}$, incubated for $5 \mathrm{~min}$ with protein block, and then washed 3 times in PBS for $5 \mathrm{~min}$. All the specimens were incubated at room temperature for $1 \mathrm{~h}$ with mouse monoclonal primary antibodies: anti-HSP90 (clone JPB24, 1:200 dilution, Leica). Sections were rinsed 3 times in PBS for $5 \mathrm{~min}$, incubated for $20 \mathrm{~min}$ with secondary antibody, and then washed 3 times in PBS for $5 \mathrm{~min}$. The detection was performed using NovoLink TM polymer (Novocastra) for $15 \mathrm{~min}$ and 3,3'-diaminobenzidine chromogen. Sections were counterstained with hematoxylin, dehydrated, and mounted. The IHC results were evaluated by two independent investigators blinded to the patients' identity and clinical status. In discrepant cases, a pathologist reviewed the cases, and a consensus was reached.

\section{Semi-quantitative and qualitative assessment of immunostaining}

Microscopal analysis using an optical microscope (Zeiss, Axioskop) was performed by two pathologists. HSP expression was evaluated according to the proportion of positively stained cells and their exact localisation. For each tumor, the percentage of immunostained cells was assessed in at least 10 optical fields under high-power magnification (G $\times 400$ ). HSP expression was evaluated according to the proportion of positively stained cells and their exact localisation. For each tumour, HSP90 staining intensities was rated on a scale of $0-3$ according to the percentage of positive tumor $(0.5 \%$ positive cells; $1,5-20 \% ; 2,20-50 \%$; or $3, .50 \%)$. The expression is very low for 0 , low for 1 , moderate for 2 and high for 3 (Figure1). HSP90 expression was classified as negative for scores $\leq 1$ and positive for scores $\geq 2$.

\section{Statistical analysis}

SPSS for Windows (17.0 SPSS, Inc. Chicago, Illinois, USA) was used for statistical analysis. Frequency tables were analyzed using the chisquare test, with Fisher's exact test to assess the significance between categorical variables. In addition, Cox regression was used to compute the hazard ratio (HR) attributed to HSP90 expression, categorized, while adjusting for age, sex, grade, loci number, tumor size, and stage. A
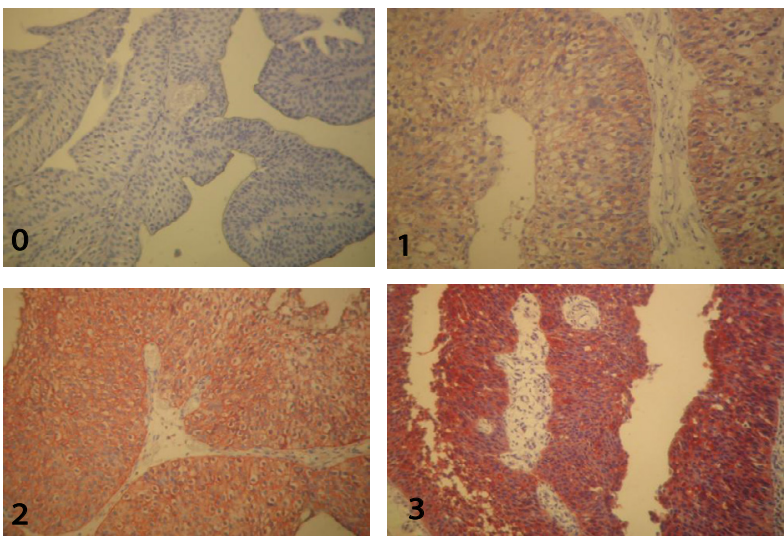

Figure 1: Non muscle invasive bladder cancer tissue illustrating the range of intensities of HSP90 immunostaining from 0 to 3. (GX200).

\begin{tabular}{|c|c|c|c|c|c|}
\hline \multirow[t]{3}{*}{ Variable } & \multicolumn{4}{|c|}{ HSP90 expression } & \multirow{3}{*}{ P Value } \\
\hline & \multicolumn{2}{|c|}{ Low } & \multicolumn{2}{|c|}{ High } & \\
\hline & $\mathrm{n} / \mathrm{N}$ & $\%$ & $\mathrm{n} / \mathrm{N}$ & $\%$ & \\
\hline \multicolumn{6}{|l|}{ Sex } \\
\hline Female & 0 & 0 & 5 & 100 & \multirow{2}{*}{0.266} \\
\hline Male & 5 & 13.2 & 33 & 86.8 & \\
\hline \multicolumn{6}{|l|}{ Age,yr } \\
\hline$<60$ & 2 & 13.3 & 13 & 86.7 & \multirow{2}{*}{0.579} \\
\hline$>=60$ & 3 & 10.7 & 25 & 89.3 & \\
\hline \multicolumn{6}{|l|}{ Tumor size } \\
\hline$<3 \mathrm{~cm}$ & 2 & 11.8 & 15 & 88.2 & \multirow{2}{*}{0.668} \\
\hline$\geq 3 \mathrm{~cm}$ & 3 & 11.5 & 23 & 88.5 & \\
\hline \multicolumn{6}{|c|}{ No.of Tumor } \\
\hline Single & 3 & 11.5 & 23 & 88.5 & \multirow{2}{*}{0.668} \\
\hline Multiple & 2 & 11.8 & 15 & 88.2 & \\
\hline \multicolumn{6}{|l|}{ Grade } \\
\hline Low grade & 3 & 10 & 27 & 90 & \multirow{2}{*}{0.482} \\
\hline High grade & 2 & 15.4 & 11 & 84.6 & \\
\hline \multicolumn{6}{|l|}{ Stage } \\
\hline pTa & 2 & 10 & 18 & 90 & \multirow{2}{*}{0.569} \\
\hline pT1 & 3 & 13 & 20 & 87 & \\
\hline
\end{tabular}

Table 1: Association between HSP90 expression in NMIBC with baseline characteristics. 
multivariate Cox regression was used to select the best predictive model based on the AIC criteria as well as some biological considerations. All reported $\mathrm{p}$ values were two-sided and statistical significance was considered as $\mathrm{p}$ less than .05 .

\section{Results}

\section{Host and tumors characteristics}

Our study included 43 patients ( 5 women and 38 men). The median age was 60 years (range, 25-80). 15 patients (34.88\%) were aged less than 60 years and $28(65.12 \%)$ were aged over 60 years. Tumors were multiple in 17 cases $(39.53 \%)$ and tumor size was more than $3 \mathrm{~cm}$ in 26 patients $(60.47 \%)$ (Table 2$)$. The 43 studied tumors were subdivided into 20 Pta stage (46.51\%) and 23 pT1 stage (53.49\%). All patients were alive at the end of the follow-up period and no patient was lost to follow-up. Patients without recurrence were 28 (65.11\%) while 15 (34.88\%) had experienced at least one recurrence.

\section{The association of HSP90 with clinicopathological Variables}

HSP90 staining mainly located in cytoplasm of tumor cells. Overexpression of HSP90 was observed in 33 of $43(76.74 \%)$ of NMIBC samples. According to the results of immunohistochemistry, we correlated HSP90 status in 43 NMIBC specimens with other widely recognized clinicopathologic parameters (Table 1). Our analyses showed that no significant association was observed between HSP90 positive expression levels and the clinicopathologics factors.

\section{Relationship between HSP90 expression and BCG immunotherapy response}

Univariate Cox regression analysis of baseline characteristics and

\begin{tabular}{|c|c|c|c|c|c|}
\hline $\begin{array}{l}\text { Predictive } \\
\text { factors }\end{array}$ & Frequency & No. evenement & HR & $\mathbf{P}$ & $95 \% \mathrm{Cl}$ \\
\hline \multicolumn{6}{|l|}{ Sex } \\
\hline Male & $38(88.37)$ & $12(31.58)$ & \multirow[t]{2}{*}{1.086} & \multirow[t]{2}{*}{0.894} & \multirow[t]{2}{*}{$0.324-3.640$} \\
\hline Female & $5(11.63)$ & $3(60)$ & & & \\
\hline \multicolumn{6}{|l|}{ Age } \\
\hline$<60$ & $15(34.88)$ & $4(26.66)$ & \multirow[t]{2}{*}{0.933} & \multirow[t]{2}{*}{0.873} & \multirow[t]{2}{*}{$0.399-2.181$} \\
\hline$\geq 60$ & $28(65.12)$ & $11(39.29)$ & & & \\
\hline \multicolumn{3}{|c|}{ Multiplicity } & & & \\
\hline Simple & $26(60.47)$ & $8(30.77)$ & \multirow{2}{*}{1.308} & \multirow{2}{*}{0.536} & \multirow{2}{*}{$0.560-3.056$} \\
\hline Multiple & $17(39.53)$ & $7(41.18)$ & & & \\
\hline \multicolumn{3}{|c|}{ Tumor size } & & & \\
\hline$<3 \mathrm{~cm}$ & $17(39.53)$ & $7(41.18)$ & \multirow{2}{*}{0.918} & \multirow{2}{*}{0.838} & \multirow{2}{*}{$0.402-2.097$} \\
\hline$\geq 3 \mathrm{~cm}$ & $26(60.47)$ & $8(30.77)$ & & & \\
\hline \multicolumn{6}{|l|}{ Stage } \\
\hline pTa & $20(46.51)$ & $6(30)$ & \multirow{2}{*}{0.821} & \multirow{2}{*}{0.635} & \multirow{2}{*}{$0.365-1.849$} \\
\hline pT1 & $23(53.49)$ & $9(39.13)$ & & & \\
\hline \multicolumn{6}{|l|}{ Grade } \\
\hline Low & $30(69.78)$ & $10(33.33)$ & \multirow{2}{*}{1.123} & \multirow{2}{*}{0.737} & \multirow{2}{*}{$0.571-2.211$} \\
\hline High & $13(30.24)$ & $5(38.46)$ & & & \\
\hline \multicolumn{3}{|c|}{ HSP90 expression } & & & \\
\hline Low & $5(11.6)$ & $3(12.5)$ & \multirow[t]{2}{*}{1.086} & \multirow[t]{2}{*}{0.894} & \multirow[t]{2}{*}{$0.324-3.640$} \\
\hline High & & $21(87.5)$ & & & \\
\hline
\end{tabular}

Abbreviations: HR: Hasard Ratio; $95 \% \mathrm{Cl}$ : Confiance Interval ; $p<0.05$ is significant.

Table 2: Univariate Cox regression analysis to predict time to recurrence following end of treatment.

\begin{tabular}{|l|c|c|c|}
\hline Variable & P & HR & $\mathbf{9 5 \%} \mathbf{C l}$ \\
\hline Sexe (F vs. M) & 0.796 & 1.200 & $0.318-3.763$ \\
\hline Age des patients $(<60$ vs $\geq 60)$ & 0.854 & 0.902 & $0.300-2.713$ \\
\hline Grade (low vs. high) & 0.991 & 1.005 & $0.421-2.398$ \\
\hline Stage (T1 vs. Ta) & 0.641 & 0.791 & $0.296-2.118$ \\
\hline Tumor size (<3 vs $\geq 3$ ) & 0.952 & 0.972 & $0.377-2.505$ \\
\hline Multiplicity & 0.504 & 1.358 & $0.554-3.331$ \\
\hline HSP90 (low vs. high) & 0.884 & 1.096 & $0.319-3.768$ \\
\hline
\end{tabular}

Abbreviations: HR: Hazard ratio; $95 \% \mathrm{Cl}$ : Confidence Interval; $p<0.05$ is significant.

Table 3: Multivariate analysis of disease recurrence in patient with NMIBC treated by BCG immunotherapy.

HSP90 expression showed that no significant association was found with BCG immunotherapy response (Table 2). In the other hand, multivariate Cox regression analysis showed that HSP90 protein is not an independent factor of tumor response after BCG immunotherapy (Table 3).

\section{Discussion}

In non-muscle invasive bladder cancer, the main problem continues to be the avoidance of infiltrative recurrence or progression without overtreatment. One of the major issues of BCG immunotherapy in NMIBC is to identify patients who could have refractory response and undergo to tumor recurrence. Currently, there are no relevant tumor characteristics correlated with a recurrence potential of these neoplasms after immunotherapy. This treatment is a standard therapy for NMIBC but its mechanism is not completely understood. Both immunological mechanisms and/or direct effects on tumor cells have been proposed, the former being more extensively studied. The direct effect on tumor cells has been less investigated in part because the immune therapy with BCG is administered after tumor resection. However, it is possible that a number of tumor cells remain in the bladder mucosa and thus the biological behavior of urothelial cancer cells could play a role in the global response to instilled BCG. Thus the identification of biomarkers that prospectively evaluate disease aggressiveness, progression risk, probability of recurrence and overall prognosis would improve patient care. At this time, the decision to preserve the bladder or to perform a cystectomy depends on a number of clinicopathologic parameters, but none are able to sufficiently identify patients for the appropriate therapeutic modality. Thus, integration of molecular markers with conventional clinicopathologic markers of NMIBC may refine clinical decision making for the selection of adjuvant therapy. In fact, a delay in appropriate therapy may lead to metastatic dissemination. Predictive factors in bladder tumours have been studied extensively; however, neither the expression of oncogenes (ras, erbB-2), nor tumour suppressor genes (p53), nor cell cycle genes (p16p15) has permitted discrimination of the BCG non responder tumours [16]. Recently, Heat shock proteins (HSPs) are found to be increased in many solid tumors and haematological malignancies.

Their expression may play an important role in maintaining protein homoeostasis of malignant cells even in the hostile hypoxic and acidotic microenvironment of the tumor. It has been reported that overexpression of HSPs is associated with poor prognosis, such as HSP27 in rectal cancer. Indeed, a study demonstrated the strong correlation between high expression of HSP27 and poor cancer-specific survival in rectal tumor $(\mathrm{n}=205, \mathrm{p}=0.0063)$. In addition, multivariate Cox regression strengthened the prognostic significance of both nodal metastases $(\mathrm{p}=0.0001)$ and HSP27 expression $(\mathrm{p}=0.0233)$ in independently predicting survival in rectal cancer [17]. In pancreatic adenocarcinoma, Ali Aghdassi et al. showed that pancreatic cancer cells expressed 
significantly higher Hsp70 levels compared with nonmalignant ductal cells, and this is important because suggests that Hsp70 plays a role in tumor cell resistance to apoptosis [18], and in gastrointestinal stromal tumors, Kang GH and al found that the Cox proportional hazards model indicated the HSP90 expression as an independent predictor of recurrence in GISTs $(P=0.003)$ [19]. Nevertheless, the role of HSP90 in NMIBC has not been thoroughly elucidated.

Currently, few data are available on the relationship between the expression of these proteins in urothelial tumors and BCG immunotherapy response. These proteins are expressed in normal urothelium and decreased expression has been detected particularly in invasive bladder transitional cell carcinoma. To the best of our knowledge, our current study is the first to investigate the relationship between HSP90 expression and the response to BCG therapy in patients having NMIBC. In the present study, we described the prognostic value of HSP90 protein expression in NMIBC and its relationship with the response to BCG immunotherapy. Immunohistochemistry was used to analyze the correlation of HSP90 protein expression with clinical pathological factors and prognostic effect of 43 NMIBC patients. The result demonstrated that HSP90 protein levels were not significantly associated with the response to BCG immunotherapy. However, other previous studies have demonstrated an important correlation between the loss of HSP90 expression and poor prognosis [10]. In the other hand, Thierry Lebret et al. [20] found a significant association between HSP90 expression and the response to BCG immunotherapy. They have demonstrated that patients who had a tumour with $<40 \%$ HSP90 expression failed to respond to BCG treatment and required cystectomy as second-line treatment. In contrast, patients with $>40 \%$ of HSP90 tumour expression, who had responded partially (no infiltrative recurrence) or totally (no recurrence) to BCG, were effectively controlled with local immunotherapy. The difference found between our results could be explained by the fact that Thierry Lebret and al evaluated only high grade NMIBC that had a different behavioural pattern as compared to all grade of NMIBC. In the other hand, Cardillo et al. [21] reported, for high-grade bladder tumours, that $92.9 \%$ of tumours expressed HSP90 but in similar to our results no correlation with prognosis was found [21]. Yu et al. [22] investigated the prognostic value of HSP27, HSP60, HSP70 and HSP90 on samples collected from 744 clinical cases. The found that High expression of HSP70 predicted recurrence of NMIBC treated by transurethral resection, and low expression of HSP27 correlated with progression and cancer-specific mortality for NMIBC treated by transurethral resection. Low expression of HSP27 also predicted cancerspecific mortality for patients who underwent cystectomy. However, HSP90 was not associated with the biological behavior of NMIBC [22].

Several limitations of this study should be considered. First, the sample size was small and follow-up was short. Second, the clinical application of immunohistochemistry is limited by its reproducibility and reliability. Conversely, several strengths of the study should be noted. Our series is well selected, which provides valuable information.

\section{Conclusion}

In summary, this study demonstrates that HSP90 could not be a useful prognostic marker for BCG treatment in NMIBC. These preliminary data warrant further studies using a larger scale of patients to provide useful prognostic information that could ultimately help to develop individualized surveillance programs.

\section{Declaration of Interest}

The authors report no conflicts of interest. The authors alone are responsible for the content and writing of the paper.

\section{References}

1. Herr HW, Wartinger DD, Fair WR, Oettgen HF (1992) Bacillus Calmette-Guerin therapy for superficial bladder cancer: a 10-year followup. J Urol 147: 10201023.

2. Sylvester RJ, van der MEIJDEN AP, Lamm DL (2002) Intravesical bacillus Calmette-Guerin reduces the risk of progression in patients with superficial bladder cancer: a meta-analysis of the published results of randomized clinical trials. J Urol 168: 1964-1970.

3. Shahin O, Thalmann GN, Rentsch C, Mazzucchelli L, Studer UE (2003) A retrospective analysis of 153 patients treated with or without intravesical bacillus Calmette-Guerin for primary stage T1 grade 3 bladder cancer: recurrence, progression and survival. J Urol 169: 96-100.

4. Lebret T, Bohin D, Kassardjian Z, Herve JM, Molinie V, et al. (2000) Recurrence, progression and success in stage Ta grade 3 bladder tumors treated with low dose bacillus Calmette-Guerin instillations. J Urol 163: 63-67.

5. Whitesell L, Lindquist SL (2005) HSP90 and the chaperoning of cancer. Nat Rev Cancer 5: 761-772.

6. Calderwood SK, Khaleque MA, Sawyer DB, Ciocca DR (2006) Heat shock proteins in cancer: chaperones of tumorigenesis. Trends Biochem Sci 31: 164172.

7. Dai $C$, Whitesell L, Rogers AB, Lindquist $S$ (2007) Heat shock factor 1 is a powerful multifaceted modifier of carcinogenesis. Cell 130: 1005-1018.

8. Min JN, Huang L, Zimonjic DB, Moskophidis D, Mivechi NF (2007) Selective suppression of lymphomas by functional loss of Hsf1 in a p53-deficient mouse model for spontaneous tumors. Oncogene 26: 5086-5097.

9. Sreedhar AS, Kalmár E, Csermely P, Shen YF (2004) Hsp90 isoforms: functions, expression and clinical importance. FEBS Lett 562: 11-15.

10. Lebret T, Watson RW, Molinié V, O'Neill A, Gabriel C, et al. (2003) Heat shock proteins HSP27, HSP60, HSP70, and HSP90: expression in bladder carcinoma. Cancer 98: 970-977.

11. Welch WJ (1992) Mammalian stress response: cell physiology, structure/ function of stress proteins, and implications for medicine and disease. Physiol Rev 72: 1063-1081.

12. Sireci G, Dieli F, Salerno A (2000) T cells recognize an immunodominant epitope of heat shock protein 65 in Kawasaki disease. Mol Med 6: 581-590.

13. Kaufmann SH (1990) Heat shock proteins and the immune response. Immunol Today 11: 129-136.

14. Lebret T, Watson RW, Fitzpatrick JM (2003) Heat shock proteins: their role in urological tumors. J Urol 169: 338-346.

15. Sauter G, Algaba F, Amin MB (2004) Non invasive urothelial tumors. In: Elbe JN, Sauter G, Epstein JI, Sesterhenn IA,eds. Pathology and Genetics: Tumors of the Urinary System and Male Genital Organs. Lyon: IARC Press.

16. Brandau S, Böhle A (2001) Bladder cancer. I. Molecular and genetic basis of carcinogenesis. Eur Urol 39: 491-497.

17. Tweedle EM, Khattak I, Ang CW, Nedjadi T, Jenkins R, et al. (2010) Low molecular weight heat shock protein HSP27 is a prognostic indicator in rectal cancer but not colon cancer. Gut 59: 1501-1510.

18. Aghdassi A, Phillips P, Dudeja V, Dhaulakhandi D, Sharif R, et al. (2007) Heat shock protein 70 increases tumorigenicity and inhibits apoptosis in pancreatic adenocarcinoma. Cancer Res 67: 616-625.

19. Kang GH, Lee EJ, Jang KT, Kim KM, Park CK, et al. (2010) Expression of HSP90 in gastrointestinal stromal tumours and mesenchymal tumours. Histopathology 56: 694-701.

20. Lebret T, Watson RW, Molinié V, Poulain JE, O'Neill A (2007) HSP90 Expression: A New Predictive Factor for BCG Response in Stage Ta-T1 Grade 3 Bladder Tumours. Eur Urol 51: 161-167.

21. Cardillo MR, Sale P, Di Silverio F (2000) Heat shock protein-90, IL-6 and IL-10 in bladder cancer. Anticancer Res 20: 4579-4583.

22. Yu HJ, Chang YH, Pan CC (2013) Prognostic significance of heat shock proteins in urothelial carcinoma of the urinary bladder. Histopathology 62: 788798. 